\title{
Test-Retest Reliability of the Balance Tracking System Modified Clinical Test of Sensory Integration and Balance Protocol Across Multiple Time Durations
}

\author{
Daniel J Goble (D) \\ Nathan O Conner' \\ Michael R Nolff ' \\ Joshua L Haworth (ID' \\ Bradley J Kendall ${ }^{2}$ \\ 'Exercise Science Program, Department \\ of Human Movement Science, Oakland \\ University, Rochester, MI, 48309, USA; \\ ${ }^{2}$ Department of Kinesiology, Taylor \\ University, Upland, IN, 46989, USA
}

Background: Postural control is critical for body sway control and is subserved by three sources of sensory feedback (ie, vision, proprioception and vestibulation). A method for determining the relative contribution of each sensory feedback source to postural control is the modified clinical test of sensory integration and balance for the balance tracking system (BTrackS). However, this method has not yet been evaluated for test-retest reliability.

Purpose: To determine the test-retest reliability of the modified clinical test of sensory integration and balance protocol for the BTrackS across multiple time intervals.

Methods: Three groups of healthy young adults performed the BTrackS modified clinical test of sensory integration and balance protocol four times separated by either one day, one week or one month. Within each time duration group, and condition, differences in total center of pressure path length were determined from one test session to the next and intra class correlation coefficient categorizations were made.

Results: In all but one case, no significant difference in performance was seen from one testing session to the next. The one significant difference found was a decrease in total center of pressure path length from day 1 to day 2 in the vestibular condition of the group tested daily. Intra class correlation coefficient results largely indicated fair-good reliability across time durations and test conditions.

Conclusion: The present study largely supports use of the BTrackS modified clinical test of sensory integration and balance protocol as a means of probing the sensory contributions to balance performance across multiple time durations.

Keywords: sensory feedback, postural control, reliability, balance assessment

\section{Introduction}

Maintaining one's balance while standing is a fundamental aspect of human behavior. This activity requires "postural control" in order to regulate body sway and keep one's center of mass over the base of support (ie, the area below and between the feet). Postural control is multifactorial in nature, involving contributions from both motor and sensory systems. With respect to the latter, three senses are thought to be significant contributors to postural control. Proprioception of the ankle gives sway-related feedback based on an inverted pendulum model of body dynamics. ${ }^{1}$ Visual information helps anticipate a loss in balance using head position
Correspondence: Daniel J Goble

Exercise Science Program, Department of Human Movement Science, Oakland University, 433 Meadow Brook Road, Rochester, MI, 48309-4452, USA

Tel +I $248364-8688$

Fax + I 248 364-8657

Email dgoble@oakland.edu 
cues relative to the surrounding environment. ${ }^{2}$ Lastly, vestibular feedback is utilized to monitor conflicts between head location and the external environment. ${ }^{3}$

Several testing protocols exist that attempt to highlight the individual contributions of the three balance-related sensory systems. The most widely utilized of these protocols is the modified Clinical Test of Sensory Integration and Balance (mCTSIB), which is a derivative of the CTSIB originally proposed by Shumway-Cook and Horak. ${ }^{4}$ In the mCTSIB, a comparison of postural control is made between various conditions that are designed to manipulate sensory feedback in a targeted fashion. Typically, this is achieved by altering the availability of vision through eyes open versus closed instructions, and/or reducing the fidelity of proprioception by having the participant stand on hard versus compliant (ie, foam) surface.

Performance on the mCTSIB originally relied on the subjective assessment of body sway characteristics by an individual administering the test. ${ }^{4}$ However, sophisticated approaches have subsequently evolved that allow objective evaluation of the mCTSIB using computerized force plate systems. Force plates are medical devices that precisely measure postural control by tracking the location of an individual's center of pressure (COP) when stood upon. COP is related to control of the body's center of mass (ie, postural sway), and increases in COP displacement are associated with increased postural sway and poorer balance ability. ${ }^{5}$

The Balance Tracking System (BTrackS) is a low-cost, portable force plate with software that implements a quick $(\sim 2$ $\mathrm{min}$ ), four-trial version of the MCTSIB protocol. The first trial is the "Standard" condition, where all three sensory systems are uncompromised (ie, testing is done with eyes open on a firm surface). The second trial represents the "Proprioception" condition, where vision is removed by closing the eyes. This manipulation increases reliance on both proprioceptive and vestibular systems, but proprioception is more heavily utilized for balance than vestibular information. ${ }^{6}$ The third trial represents the "Vision" condition, where reliability of the proprioceptive system is manipulated by having the individual being tested stand on a foam cushion. In this case, vision is the dominant sense remaining uncompromised over vestibulation. ${ }^{7}$ Lastly, the fourth trial represents the "Vestibular" condition, where vision is removed (ie, eyes closed) and proprioception is rendered unreliable with a foam cushion. This situation causes a shift to reliance on the uncompromised vestibular system.
While previous studies of the BTrackS mCTSIB have focused on providing normative data, there is no published work addressing the test-retest reliability of this protocol. $^{8,9}$ This includes reliability across common testing time intervals such as day to day (D2D), week to week (W2W) and month to month (M2M). The purpose of the present study was, therefore, to determine the test-retest reliability of the BTrackS mCTSIB across multiple time durations. This was accomplished by recording results from three groups of participants over four testing sessions that differed in terms of time between tests (ie, D2D, $\mathrm{W} 2 \mathrm{~W}$ or M2M). Overall, it was hypothesized that the BTrackS mCTSIB would have acceptable (ie, fair to good) test-retest reliability, supporting its clinical use across the specified time durations.

\section{Materials and Methods \\ Participants}

The sample for this study was 90 young adults separated into three equal $(\mathrm{n}=30)$ time duration groups $(\mathrm{D} 2 \mathrm{D}$, $\mathrm{W} 2 \mathrm{~W}$ and $\mathrm{M} 2 \mathrm{M}$ ). Group size was based on a power analysis of pilot results, and each group was generally well matched for sex and age characteristics as shown in Table 1. Participants self-identified as being in good general health and without any known balance issues at the time of each testing session. Ethical approval for this research was obtained from the Institutional Review Board of Oakland University in accordance with the Declaration of Helsinki. Participants provided written informed consent prior to testing.

\section{Equipment and Procedures}

The primary equipment used in this study consisted of the BTrackS Balance Plate and a laptop running the BTrackS Assess Balance Advanced software program (Figure 1). The dimensions of the BTrackS Balance Plate are $0.4 \times 0.6 \mathrm{~m}$ and it is registered as a medical device with the United States Food and Drug Administration. Due to its minimalistic design, the plate is lightweight $(<7 \mathrm{~kg})$ and easily portable. Previous studies evaluating the ability of BTrackS to collect

Table I Sex and Age Characteristics for Time Duration Groups

\begin{tabular}{|l|c|c|}
\hline & Mean \pm SD Age (Years) & Male/Female Ratio \\
\hline D2D & $24.5 \pm 2.0$ & $13 / 17$ \\
W2W & $23.6 \pm 2.9$ & $12 / 18$ \\
M2M & $24.2 \pm 4.8$ & $14 / 16$ \\
\hline
\end{tabular}

Abbreviations: D2D, day to day; W2W, week to week; M2M, month to month. 


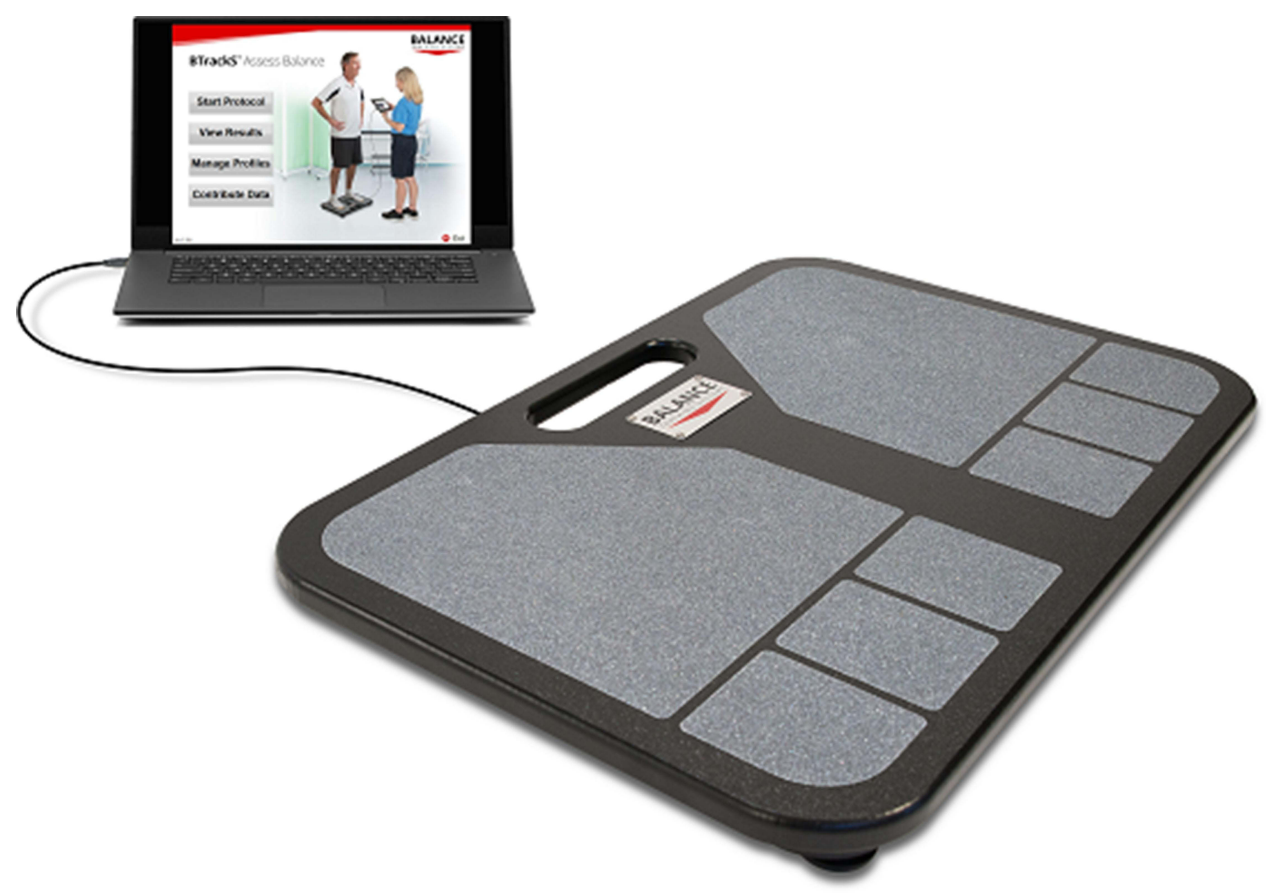

Figure I Experimental equipment used in this study included the BTrackS Balance Plate (right) and BTrackS Assess Balance software running on a laptop (left). Image provided is property of corresponding author.

COP data have found it to be ecologically valid, highly accurate, and precise. ${ }^{10-13}$ For some conditions in this study, participants were also asked to stand on a lightweight, high-density foam cushion provided with the BTrackS Balance Plate and software. The top surface of the foam cushion was $0.5 \times 0.4 \mathrm{~m}$ and the height was $6 \mathrm{~cm}$.

Participants completed four testing sessions separated by either one day (D2D group), one week (W2W group) or one month (M2M group). These sessions occurred in an isolated space with limited distractions and were guided by the BTrackS Assess Balance Advanced software. Specifically, the software provided onscreen instructions to assist the test administrator in creating participant profiles, collecting COP data, and determining BTrackS mCTSIB results. The user-friendliness of the software interface allowed administrators to achieve testing competency with minimal training. In this case, test administrators became proficient within several practice sessions performed under the guidance of an experienced user.

To start each testing session, the BTrackS Balance Plate was placed on a hard surface and levelled using built-in, height adjustable legs. The BTrackS Balance Plate was then connected to the laptop via a USB interface, which also provided power to the plate. Standardized instructions were read to participants according to the following on-screen script:
You are about to perform a modified Clinical Test of Sensory Integration and Balance or mCTSIB. The mCTSIB consists of four, 20-second trials that measure your ability to control body sway when sensory feedback is systematically manipulated. For each trial, you will stand as still as possible on the BTrackS Balance Plate with your hands on your hips and feet shoulder width apart. You will hear a tone at the beginning and end of each trial. Your mCTSIB results will be based on the Center of Pressure Path Length from the forces you place on the BTrackS Balance Plate during standing. Sensory feedback will be manipulated by having you close your eyes or stand on foam in some conditions.

In accordance with the above instructions, participants stood with feet shoulder width apart on the BTrackS Balance Plate for four consecutive 20-s testing trials (Figure 2). Participants were tested without shoes, although research has shown footwear does not impact mCTSIB results. ${ }^{14}$ Each trial began and ended with an auditory tone and had the participant place his or her hands on their hips. The first trial (ie, Standard condition, Figure 2A) required the participant to open their eyes while standing on the firm surface of the plate. After a short inter-trial delay, the second trial (ie, Proprioception condition, Figure 2B) commenced where participants had their eyes closed while standing on the 
A

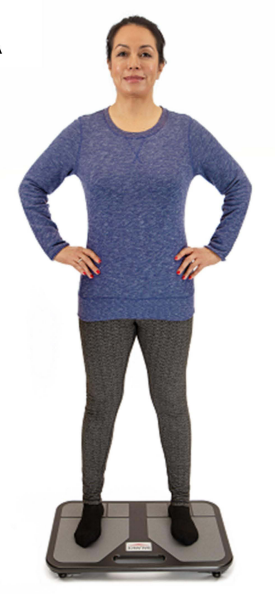

C

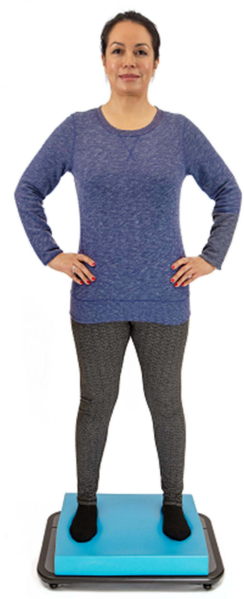

B

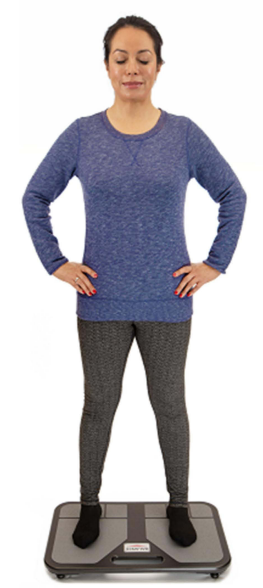

D

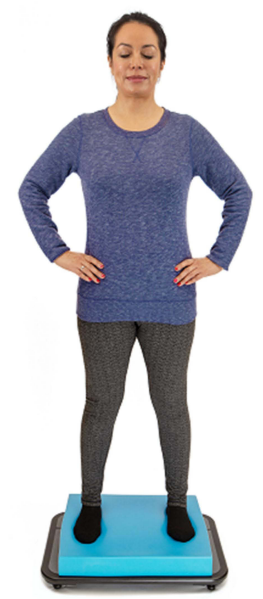

Figure 2 The four testing trial conditions utilized in the BTrackS mCTSIB protocol. Each trial requires individuals to stand as still as possible on the BTrackS Balance Plate with feet shoulder width and hands on hips. Trial I ((A) Standard condition) is performed with eyes open while standing on the firm plate surface. Trial 2 ((B) Proprioceptive condition) is performed with eyes closed while standing on the firm plate surface. Trial 3 ((C) Vision condition) and trial 4 ((D) Vestibular condition) with eyes open and closed, respectively, while standing on a compliant foam cushion. The individual depicted in this figure was not a study participant and gave informed consent for use of her likeness.

firm plate's surface. Trials three (ie, Vision condition, Figure 2C) and four (ie, Vestibular condition, Figure 2D) were done in a similar fashion to trials one and two, respectively. In this case, however, participants stood on a compliant foam cushion during testing, which was placed on top of the BTrackS Balance Plate. Trial three had the participants' eyes open while standing on the foam, while trial four had the participants' close their eyes while on the foam.

\section{Data Analysis}

Results for each trial (ie, sensory condition) were calculated and outputted by the BTrackS Assess Balance

Advanced software. BTrackS mCTSIB results were based on the Total COP Path Length, which is the default metric outputted by the system and a proxy for the magnitude of body sway. Total COP Path Length was determined by first quantifying the point-to-point COP Path Length between successive time points according to the following formula:

COP Path Length $=\left(\left(\mathrm{COP}_{\mathrm{x} 2}-\mathrm{COP}_{\mathrm{x} 1}\right)^{2}+\left(\mathrm{COP}_{\mathrm{y} 2}-\mathrm{COP}_{\mathrm{y} 1}\right)^{2}\right)^{0.5}$

where, $\mathrm{COP}_{\mathrm{x} 2}$ and $\mathrm{COP}_{\mathrm{x} 1}$ are adjacent time points in the $\mathrm{COP}_{\mathrm{x}}$ (medial/lateral) time series and $\mathrm{COP}_{\mathrm{y} 2}$ and $\mathrm{COP}_{\mathrm{y} 1}$ are adjacent time points in the $\mathrm{COP}_{\mathrm{y}}$ (anterior/posterior) time series. The sum of all COP Path Lengths across a trial was then added together to get Total COP Path Length.

For each time duration group (ie, D2D, W2W, M2M) and mCTSIB condition (ie, Standard, Proprioception, Vision, Vestibular) differences in Total COP Path Length from one test session to the next were determined using paired, two-tailed t-tests. Significance was considered at the $p<0.001$ level based on a Bonferroni correction of $p<$ $0.05 / 36=0.001$ to control for multiple comparisons. Where significant effects were found Cohen's d was calculated as a measure of effect size. The test-retest reliability of each mCTSIB condition was also quantified across all sessions using an intra-class correlation coefficient (ICC) model with two-way mixed-effects. Categories for ICCs results were: $0.00-0.39=$ Poor, $0.40-0.59=$ Fair, $0.60-0.74=$ Good and $0.75-1.00=$ Excellent $^{15}$

\section{Results}

The mean \pm SD Total COP Path Length for the D2D, W2W and M2M groups in each BTrackS mCTSIB condition (ie, Standard, Proprioception, Vision, Vestibular) is shown in Figure 3. In almost all cases, the comparison from one testing session to the next had no significant difference in Total COP Path Length $(p>0.001)$. That said, one significant difference was found for the D2D group in the most difficult, vestibular condition. Specifically, when asked to stand on foam with eyes closed, participants had lower Total COP Path Lengths on Day 2 compared to Day $1(p<0.001$; Cohen's $\mathrm{d}=0.5)$.

ICC results quantifying the test-retest reliability of the BTrackS mCTSIB are shown in Table 2. For the D2D group, ICC values ranged from 0.47 (Fair) to 0.79 (Excellent) across the four sensory conditions. In contrast, the range of ICC values for the W2W group was only 0.46 (Fair) to 0.67 (Good) across sensory conditions. The M2M 

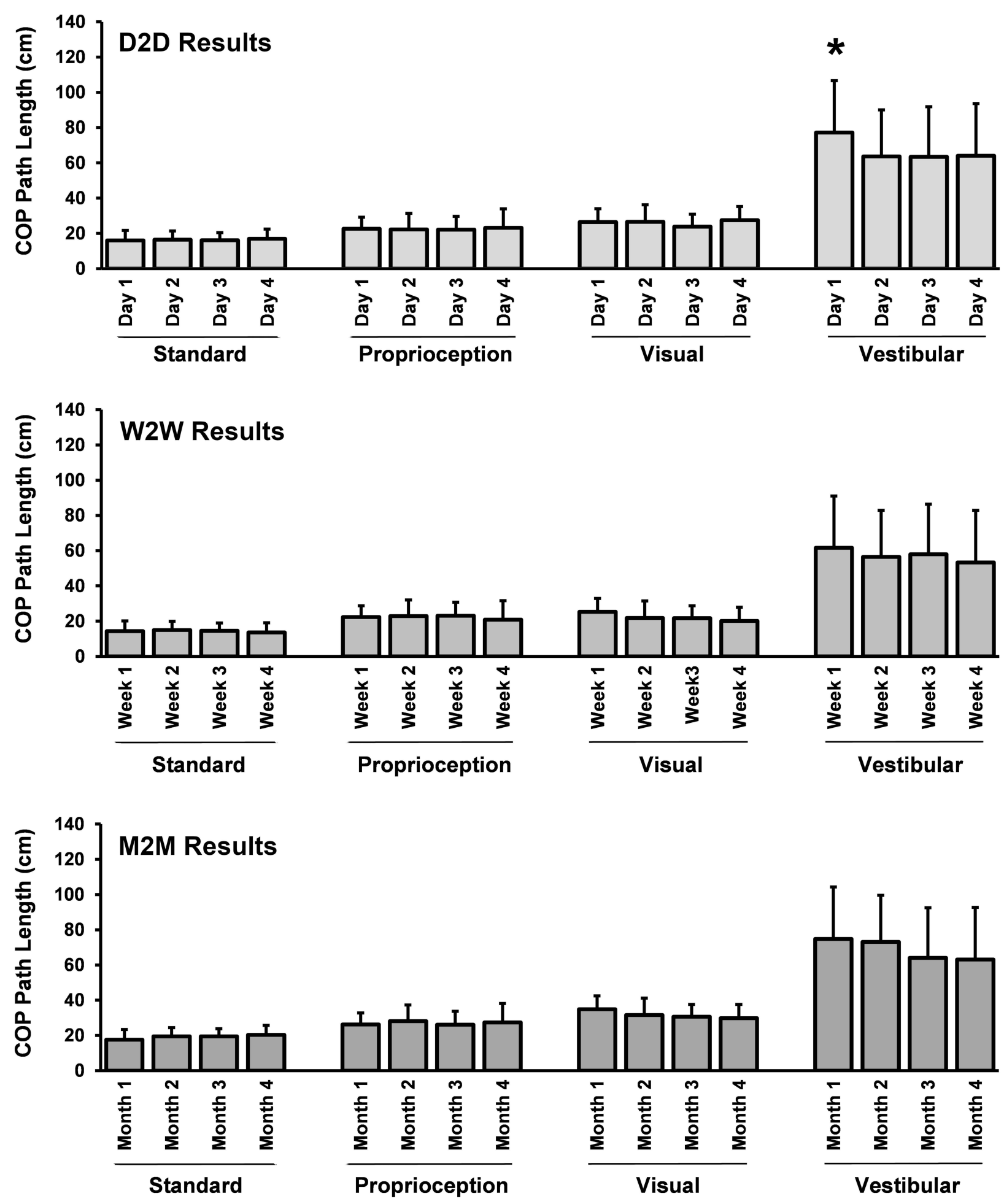

Figure 3 Mean \pm SD Total COP Path Length results for the D2D (top), W2W (middle) and M2M (bottom) time duration groups in each of the four BTrackS mCTSIB conditions (ie, Standard, Proprioception, Vision and Vestibular). A significant difference $(*)$ was only seen between Day I and Day 2 for the D2D group in the vestibular condition.

Abbreviations: COP, center of pressure; D2D, day to day; M2M, month to month; W2W, week to week. 
Table 2 Reliability of BTrackS mCTSIB Conditions Across Various Time Durations

\begin{tabular}{|l|c|c|c|}
\hline \multirow{2}{*}{ mCTSIB Condition } & \multicolumn{3}{|c|}{ Test-Retest Reliability ICC (Category) } \\
\cline { 2 - 4 } & D2D Group & W2W Group & M2M Group \\
\hline Standard & 0.63 (Good) & 0.47 (Fair) & 0.68 (Good) \\
Proprioception & 0.69 (Good) & 0.67 (Good) & 0.59 (Fair) \\
Vision & 0.47 (Fair) & 0.46 (Fair) & 0.65 (Good) \\
Vestibular & 0.79 (Excellent) & 0.53 (Fair) & 0.48 (Fair) \\
\hline
\end{tabular}

Abbreviations: D2D, day to day; W2W, week to week; M2M, month to month; ICC, intraclass correlation coefficient.

group was similar to the $\mathrm{W} 2 \mathrm{~W}$ group with ICC values ranging from 0.48 (Fair) to 0.68 (Good) across sensory conditions. No sensory condition showed Poor test-retest reliability for any of the D2D, W2W or M2M groups.

\section{Discussion}

The present study sought to examine the test-retest reliability of the BTrackS mCTSIB protocol. Three groups of healthy adults were tested over four sessions separated by either one day (ie, D2D), one month (ie, M2M) or one week (ie, W2W). Session to session comparisons showed no significant differences in performance, except for the vestibular condition in the D2D group. Across groups and sensory conditions, ICC values were largely in the Fair to Good range with Excellent reliability seen for the D2D vestibular condition. Taken together, these results suggest that the BTrackS mCTSIB has acceptable test-retest reliability to be efficacious in typical clinical use case scenarios. However, some caution should be taken when interpreting group results in the vestibular condition over a D2D timeframe.

A lack of significant performance differences from one testing session to the next suggests that the BTrackS mCTSIB is reliable at the group level across multiple time durations. Only one significant difference was seen, whereby the D2D group had less Total COP Path Length (ie, less postural sway) on Day 2 compared to Day 1. This result may be due to a practice effect, such that recent (ie, one day) experience on the more difficult vestibular condition assisted participants in dealing with its simultaneous visual (ie, eyes closed) and vestibular (ie, foam surface) perturbations. Similar results have been shown for another balance protocol - the Balance Error Scoring System (BESS). In the BESS, more difficult testing conditions, and shorter durations between sessions, have also resulted in lower test-retest reliability. ${ }^{16,17}$

While other BTrackS protocols have shown Excellent test-retest reliability, the ICC results for the BTrackS
mCTSIB were largely in the Fair to Good range, with only one result (ie, D2D vestibular) showing Excellent test-retest reliability. ${ }^{18,19}$ These findings are in good alignment with several previous studies exploring the test-retest reliability of the Biodex force plate mCTSIB protocol. Specifically, a comparison of two same-day Biodex mCTSIB sessions found healthy young adult data had Fair to Excellent test-retest reliability for the "duration" and "stability" metrics. ${ }^{20}$ A second study of Biodex mCTSIB test-retest reliability employed two tests conducted one week apart with community dwelling women over the age of 65 years. ${ }^{21}$ The cumulative ICC result for this study was within the fair range of the present study.

BTrackS mCTSIB test-retest reliability was determined for young adults that were in good general health with no known balance issues. The positive results of this work set the stage for future work exploring a broader sample of individuals across the lifespan who may or may not have known balance impairments. Additionally, based on the likely practice effect seen for the vestibular condition in the shortest (ie, D2D) time duration, it would seem valuable for future work to explore even shorter inter-test durations such as hour to hour. Indeed, further reducing the inter-test duration may produce results that are even more prone to practice effects.

\section{Conclusion}

The results of the present study support using the BTrackS mCTSIB as a relatively reliable means of probing sensory contributions to balance performance across multiple time durations. However, care should be taken when interpreting a vestibular condition result if taken over a D2D duration, as this may be prone to a practice effect. To this point, inclusion of a practice trial for the vestibular condition might be recommended in a future version of the protocol. This would allow an opportunity for the 
participant to accommodate to the difficulty associated with eyes closed standing on a compliant foam surface.

\section{Disclosure}

DJG receives royalties paid to San Diego State University from a patent (US Patent 10660558) related to the balance technology in this study. In addition, he has an equity stake (ie, stock options) and performs contract work for Balance Tracking Systems Inc., the parent company of the BTrackS Balance Plate and BTrackS Assess Balance Advance software. This conflict is mitigated by a management plan put in place by his academic institution (Oakland University) to ensure the integrity of his research. The other authors of this work (MN, NC, $\mathrm{BK}, \mathrm{JH})$ have no conflicts of interest to declare.

\section{References}

1. Nashner LM, Shupert CL, Horak FB, et al. Organization of posture controls: an analysis of sensory and mechanical constraints. Prog Brain Res. 1989;90:411-418.

2. Black FO, Wall C, Nasher LM. Effects of visual and support surface orientation references upon postural control in vestibular deficient subjects. Acta Otolaryngol. 1983;95:199-201. doi:10.3109/ 00016488309130936

3. Nasher LM, Black FO, Wall C. Adaptation to altered support and visual conditions during stance: patients with vestibular deficits. $J \quad$ Neurosci. 1982;2:536-544. doi:10.1523/JNEUROSCI.02-0500536.1982

4. Shumway-Cook A, Horak FB. Assessing the influence of sensory interaction on balance: suggestion from the field. Phys Ther. 1986;66:1548-1550. doi:10.1093/ptj/66.10.1548

5. Richmond SB, Fling BW, Lee H, et al. The assessment of center of mass and center of pressure during quiet stance: current applications and future directions. J Biomech. 2021;123:110485. doi:10.1016/j. jbiomech.2021.110485

6. Lord SR, Clark RD, Webster IW. Postural stability an associated physiological factors in a population of aged persons. J Gerontol. 1991;46:M69-M76.

7. Simoneau GG, Leibowitz HW, Ulbrecht JS, et al. The effects of visual factors and head orientation on postural steadiness in women 55 to 70 years of age. J Gerontol. 1992;47:M151-M158. doi:10.1093/geronj/ 47.5.M151

8. Goble DJ, Brar H, Brown EC, et al. Normative data for the balance tracking system modified clinical test of sensory integration and balance protocol. Med Devices. 2019;12:183-191. doi:10.2147/MDER. S206530

Medical Devices: Evidence and Research

Publish your work in this journal

Medical Devices: Evidence and Research is an international, peerreviewed, open access journal that focuses on the evidence, technology, research, and expert opinion supporting the use and application of medical devices in the diagnosis, monitoring, treatment and management of clinical conditions and physiological processes. The identification of novel devices and optimal use of existing devices
9. Goble DJ, Brown EC, Marks CR, et al. Expanded normative data for the balance tracking system modified clinical test of sensory integration and balance protocol. Med Devices. 2020;12:e10084.

10. Levy SS, Thralls KJ, Kviatkovsky SA. Validity and reliability of a portable balance tracking system, BTrackS, in older adults. J Geriatr Phys Ther. 2018;41:102-107. doi:10.1519/ JPT.0000000000000111

11. Goble DJ, Khan E, Baweja HS, et al. A point of application study to determine the accuracy, precision and reliability of a low-cost balance plate for center of pressure measurement. $J$ Biomech. 2018;71:277-280. doi:10.1016/j.jbiomech.2018.01.040

12. O'Connor SM, Baweja HS, Goble DJ. Validating the BTrackS balance plate as a low cost alternative for the measurement of sway-induced center of pressure. $J$ Biomech. 2016;49 (16):4142-4145. doi:10.1016/j.jbiomech.2016.10.020

13. Richmond SB, Dames KD, Goble DJ, Fling BW. Leveling the playing field: evaluation of a portable instrument for quantifying balance performance. $J$ Biomech. 2018;75:102-107. doi:10.1016/j. jbiomech.2018.05.008

14. Whitney SL, Wrisley DM. The influence of footwear on timed balance scores of the modified clinical test of sensory interaction and balance. Arch Phys Med. 2004;85:439-443. doi:10.1016/j. apmr.2003.05.005

15. Cicchetti DV, Sparrow SA. Developing criteria for establishing interrater reliability of specific items: applications to assessment of adaptive behavior. Am J Ment Defic. 1981;86:127-137.

16. Broglio SP, Zhu W, Sopiarz K, et al. Generalizability theory analysis of balance error scoring system reliability in healthy young adults. J Athl Train. 2009;44(5):497-502. doi:10.4085/1062-6050-44.5.497

17. Valovich TC, Perrin DH, Gansneder BM. Repeat administration elicits a practice effect with the balance error scoring system but not with the standardized assessment of concussion in high school athletes. J Athl Train. 2003;38:51-56.

18. Haworth J, Goble D, Pile M, et al. BTrackS limits of stability test is a reliable assessment of volitional dynamic postural control. Gait Posture. 2020;80:298-301. doi:10.1016/j.gaitpost.2020.06.024

19. Hearn MC, Levy SS, Baweja HS, et al. BTrackS balance test for concussion management is resistant to practice effects. Clin J Sport Med. 2018;28(2):177-199. doi:10.1097/JSM.0000000000000435

20. Antoniadou E, Kalivioti X, Stolakis K, et al. Reliability and validity of the mCTSIB dynamic platform test to assess balance in a population of older women living in the community. J Musculoskelet Neuronal Interact. 2020;20:185-193.

21. Dawson N, Dzurrino D, Karleskint M, et al. Examining the reliability, correlation, and validity of commonly used assessment tools to measure balance. Health Sci Rep. 2018;1:e98. doi:10.1002/hsr2.98

\section{Dovepress}

which will lead to improved clinical outcomes and more effective patient management and safety is a key feature of the journal. The manuscript management system is completely online and includes a very quick and fair peer-review system. Visit http:// www.dovepress.com/testimonials.php to read real quotes from published authors. 